\title{
SIMPLE UNIVERSAL NONLINEAR LONGITUDINAL FLIGHT SIMULATION WITH AVOIDING OF STATIC AND DYNAMIC STABILITY DERIVATIVES
}

\author{
Jiří Matějů ${ }^{1}$ \\ ${ }^{1}$ Institute of Aerospace Engineering \\ Brno University of Technology \\ Technická 2, 61669 Brno, Czech Republic \\ mateju@fme.vutbr.cz
}

Keywords: Longitudinal, simulation, simple method, dynamic stability derivatives avoiding

\begin{abstract}
This paper shows simple method to simulate nonlinear longitudinal flight dynamic of an aircraft in the most direct way. The method is based on physical principles of an analytical-empiric flight dynamics. Non-linearity, as stall or thrust dependent on velocity, can be included. Static and dynamic stability derivatives are not required but can be computed as an output. The model is applicable for various aircraft conceptions. Higher level model, for example, based on flight tests data, can be modified by low-level analytical methods, e.g. for modification of horizontal tail area. No special simulation software is necessary. The model is compared to linear model and flight test experiment. This model, together with valuable analytical-empiric data, might be applied for fast flight dynamic computations. Model is easily accessible and understandable even with basic knowledge of flight dynamic and computer programming. The main application of the method is conceptual design when high precision is not expected, even if VLM, CFD or flight test data can improve the precision.
\end{abstract}

\section{INTRODUCTION}

Flight dynamic has been solved more than 100 years [1]. Classic analytical-empiric theory [2] is very useful tool for an aircraft design. Disadvantage is the time-consuming design process. In the time of high-performance computers, the simulation methods of flight dynamic [3] offer faster solution. However, combination of classic analytical methods, in its derived form, with simulation can significantly slow down the process. Non-dimensional, dimensional stability and control derivatives and derivatives in normal form are necessary to estimate [4], [5], [6], [7]. This means 3 equations for each of at least 15 stability and control derivatives for longitudinal simulation. This complexity means time-consuming work with high danger of making mistakes and problems with modification of the model. Classic linear computation has a lot of limitations and simplifications. It is usually valid only for classic conception and in close surrounding of trimmed flight. Non-linearities, as thrust and stall, are not easy to include. Drag and z component are usually neglected. Rotation of the forces from the local velocity vector to flight path a. s. is neglected. The only advantage is analytical solution.

\section{FROM CLASSIC LINEAR THEORY TO LINEAR SIMULATION}

Classic analytical-empiric linear aircraft computation requires estimation of stability and control derivatives. For example, horizontal tail influence on the aircraft flight dynamics can be expressed by following equations (do not read it, see only the complexity). 


$$
\begin{aligned}
& Z_{H T}=-p_{D} \cdot S \cdot\left(a_{H T} \cdot k_{H T} \frac{S_{V O P}}{S} \cdot\left(\varphi_{H T}^{*}-\varepsilon_{0}\right)+a_{H T} \cdot k_{H T} \cdot \frac{S_{H T}}{S} \cdot\left(1-\frac{\partial \varepsilon}{\partial \alpha}\right) \cdot\left(\alpha+\varphi_{K T}\right)-\frac{2}{c_{A}} a_{H T} \cdot\right. \\
& \left.k_{H T} \frac{S_{H T}}{S}\left(q \frac{\tilde{l}_{H T}}{V}\right)+2 a_{H T} k_{H T} \frac{S_{H T}}{S} \frac{\tilde{l}_{H T}}{c_{A}} \frac{\partial \varepsilon}{\partial \alpha}\left(\frac{\dot{\alpha} c_{A}}{2 V}\right)+a_{H T} \cdot k_{H T} \frac{S_{H T}}{S} \cdot\left(\frac{\partial \alpha}{\partial \delta}\right)_{e} \delta\right) \\
& M_{H T}=p_{D} \cdot S \cdot c_{A} \cdot\left(-a_{H T} \cdot k_{H T} \frac{S_{H T}}{S} \frac{\tilde{l}_{H T}}{c_{A}} \cdot\left(\varphi_{H T}^{*}-\varepsilon_{0}\right)-a_{H T} \cdot k_{H T} \frac{S_{H T}}{S} \frac{\tilde{l}_{H T}}{c_{A}} \cdot\left(1-\frac{\partial \varepsilon}{\partial \alpha}\right) \cdot\left(\alpha+\varphi_{K T}\right)-\right. \\
& \left.\frac{2}{c_{A}} a_{H T} \cdot k_{H T} \frac{S_{H T}}{S} \frac{\tilde{l}_{H T}}{c_{A}}\left(q \frac{\tilde{l}_{H T}}{V}\right)-2 \cdot a_{H T} \cdot k_{H T} \frac{S_{H T}}{S} \frac{\tilde{l}_{H T}}{c_{A}} \frac{\partial \varepsilon}{\partial \alpha} \frac{\tilde{l}_{H T}}{c_{A}}\left(\frac{\dot{\alpha} c_{A}}{2 V}\right)-a_{H T} \cdot k_{H T} \frac{S_{H T}}{S} \frac{\tilde{l}_{H T}}{c_{A}} \cdot\left(\frac{\partial \alpha}{\partial \delta}\right)_{e} \delta\right)
\end{aligned}
$$

After derivation of these equations by $\alpha, \overline{\dot{\alpha}}, \bar{q}, \delta$, we obtain horizontal tail part of 8 stability and control derivatives. Each of the derivative is usually composed of wing, fuselage, tail and thrust part. Even if derivatives are very useful for comparison of different aircrafts and elimination of some parameters, the simplicity and clarity of the model can be more important. In case of using basic physics combined with simulation, we can write the same equations for horizontal tail in very simple way. There is no missing information in comparison to previous equations. Body correction constant $K_{B}$ is added only.

$Z_{B H T}=-k_{\mathrm{HT}} p_{D} S_{H T} \cdot a_{\mathrm{HT}}\left(\alpha+\varphi_{\mathrm{HT}}-\varepsilon+K_{B} q \frac{\tilde{l}_{H T}}{\mathrm{~V}_{\infty}}+\left(\frac{\partial \alpha}{\partial \delta}\right)_{e} \delta\right) \quad M_{B H T}=Z_{A B H T} \tilde{l}_{H T}$

For complete simple longitudinal model of classic piston engine aircraft with simplifications mentioned above we need to add only several following equations:

$$
\begin{array}{lll}
p_{D}=\frac{1}{2} \cdot \rho \cdot V^{2} & \left.\varepsilon=\frac{2 \cdot a_{W} \cdot\left(\alpha+\varphi_{W B}-\dot{\tilde{a}_{H T}} V\right.}{V}\right) & x_{W B}=x_{W}+K_{W} K_{A B} \frac{b_{B} c_{W r}^{2}}{S} \\
Z_{W B}=-p_{D} S \cdot a_{W}\left(\alpha+\varphi_{W B}\right) & M_{W B}=p_{D} S c_{A} \cdot C_{m 0, W}-Z_{W B} x_{W B} \\
X_{W B H T}=-C_{D 0} p_{D} S-\frac{\left(Z_{W B}+Z_{B H T}\right)^{2}}{\pi A e S p_{D}} \quad X_{T}=-\frac{P_{a}}{V} & X_{G}=-m g \sin (\gamma) \quad Z_{G}=m g \cos (\gamma) \\
X=X_{W B H T}+X_{T}+X_{G} & Z=Z_{W B}+Z_{B H T}+Z_{G} & M=M_{W B}+M_{B H T} \\
\text { Trimming } \alpha \text { for } \mathrm{t}=0: \quad P_{a_{i+1}}=-X_{i} \cdot V_{i}+P_{a_{i}} & \delta_{i+1}=\delta_{i}+\frac{M_{i} / \tilde{l}_{H T}}{k_{H T} p_{D i} S_{H T} a_{H T}\left(\frac{\partial \alpha}{\partial \delta}\right)_{e}} \\
\text { Simulation: } \dot{V}=\frac{X}{m} & V_{t+\delta t}=V_{t}+\dot{V} \cdot \delta t & \dot{\gamma}=-\frac{1}{V} \frac{Z}{m} \quad \dot{q}=\frac{M}{I_{y}} \quad \dot{\alpha}=q-\dot{\gamma} \\
\Theta_{t+\delta t}=\Theta_{t}+q \cdot \delta t & \gamma_{t+\delta t}=\gamma_{t}+\dot{\gamma} \cdot \delta t & \alpha=\Theta-\gamma \quad \delta_{t}=\delta_{t=0}+\Delta \delta_{t}
\end{array}
$$

All the input parameters can be determined from aerodynamic analysis of the wing, body, horizontal tail and elevator and from mass and geometry analysis of an aircraft. Even if the model is very simple, experiment comparison at the end of this study shows, that it is close to measurement. In comparison to up to hundreds of the equations in the classic theory, which express the same model, it is high time save, even if simple solver and trimming algorithm is necessary to use.

\section{NON-LINEAR MODEL}

By using basic physics, we got only several equations. We can, therefore, afford level higher model in comparison to linear model. Rotation of the forces to the flight-path axis system is possible, $z$ component as well as drag force can be included and non-linear coefficients can be used also. Every part of an aircraft can be defined by the position and by the forces which act on it. The aerodynamic forces are computed from airflow model and aerodynamic coefficients, estimated from VLM or CFD [8], [9]. Each of the model input characteristics can be linear, non-linear function or table of values. Avoiding of most of dynamic stability derivatives was possible due to modification of an angle of attack by angular velocity and by delayed downwash model of the wing. A transformation matrix is used to 
move automatically the characteristics to the center of gravity and body fixed a. s. Trimmed flight is estimated by bisection method modified by interval expansion. The simulation is compared to Cessna 172 SP flight tests.

\subsection{Axis systems and notation}

Axis system is defined by an origin point and x-axis angle. Upper index of a symbols describes used axis system. Lower index describes a part of an aircraft.

$$
\text { Symbol part } \text { coord. system }
$$

Axis system origin symbol is omitted in forces symbols as the forces are independent of its origin. Axis system angle in moment symbols is omitted as the moment is independent of its angular displacement.

Table 1: The most used axis systems. (Red symbols, $C G$ and $\Theta$ are usually omitted for simpler notation).

\begin{tabular}{lcccccc}
\hline Axis system & Origin & x direction & Force & Moment & $\begin{array}{c}\text { Position } \\
\text { in given a. s. }\end{array}$ & $\begin{array}{c}\text { Ref-point } \\
\text { position }\end{array}$ \\
\hline $\begin{array}{l}\text { Local air-path } \\
\text { (moving air) }\end{array}$ & $\mathbf{A}_{\mathbf{j}}$ & $\vec{V}_{j}$ & $D, L$ & $M_{j}^{A_{j}}$ & $x_{j}^{A_{j}, \alpha_{j}}, z_{j}^{A_{j}, \alpha_{j}}$ & $x_{A_{j}}^{r e f}, z_{A_{j}}^{r e f}$ \\
Local body fixed & $\mathbf{F}_{\mathbf{j}}$ & $\varphi_{j}$ & $X_{j}^{\varphi_{j}}, Z_{j}^{\varphi_{j}}$ & $M_{j}^{F_{j}}$ & $x_{j}^{F_{j}, \varphi_{j}}, z_{j}^{F_{j}, \varphi_{j}}$ & $x_{F_{j}}^{r e f}, z_{F_{j}}^{r e f}$ \\
Body & $\mathbf{C G}$ & $\Theta$ & $X_{j}^{\Theta}, Z_{j}^{\Theta}$ & $M^{C G}$ & $x^{C G, \Theta}, z^{C G, \Theta}$ & $x_{C G}^{r e f}, z_{C G}^{r e f}$ \\
Flight path & $\mathbf{C G}$ & $\gamma$ & $X_{j}^{\gamma}, Z_{j}^{\gamma}$ & $M^{C G}$ & $x^{C G, \gamma, z^{C G, \gamma}}$ & $x_{C G}^{r e f}, z_{C G}^{r e f}$ \\
Earth & $\mathbf{C G}$ & $\gamma=0, x \perp \vec{g}$ & $\boldsymbol{X}^{g}, Z^{g}$ & $M^{C G}$ & $x^{C G, g}, z^{C G, g}$ & $x_{C G}^{r e f}, z_{C G}^{r e f}$ \\
Reference & Ref. & $\Theta+180^{\circ}$ & & & $x_{j}^{r e f}, z_{j}^{r e f}$ & \\
\hline
\end{tabular}

x-axis of local air-path a. s. has a direction of air velocity vector. $x$-axis of local body system has a direction of incidence angle at given position, for example propeller revolution axis. If the symbol in upper index is missing, it means $\Theta$ orientation and $C G$ origin.

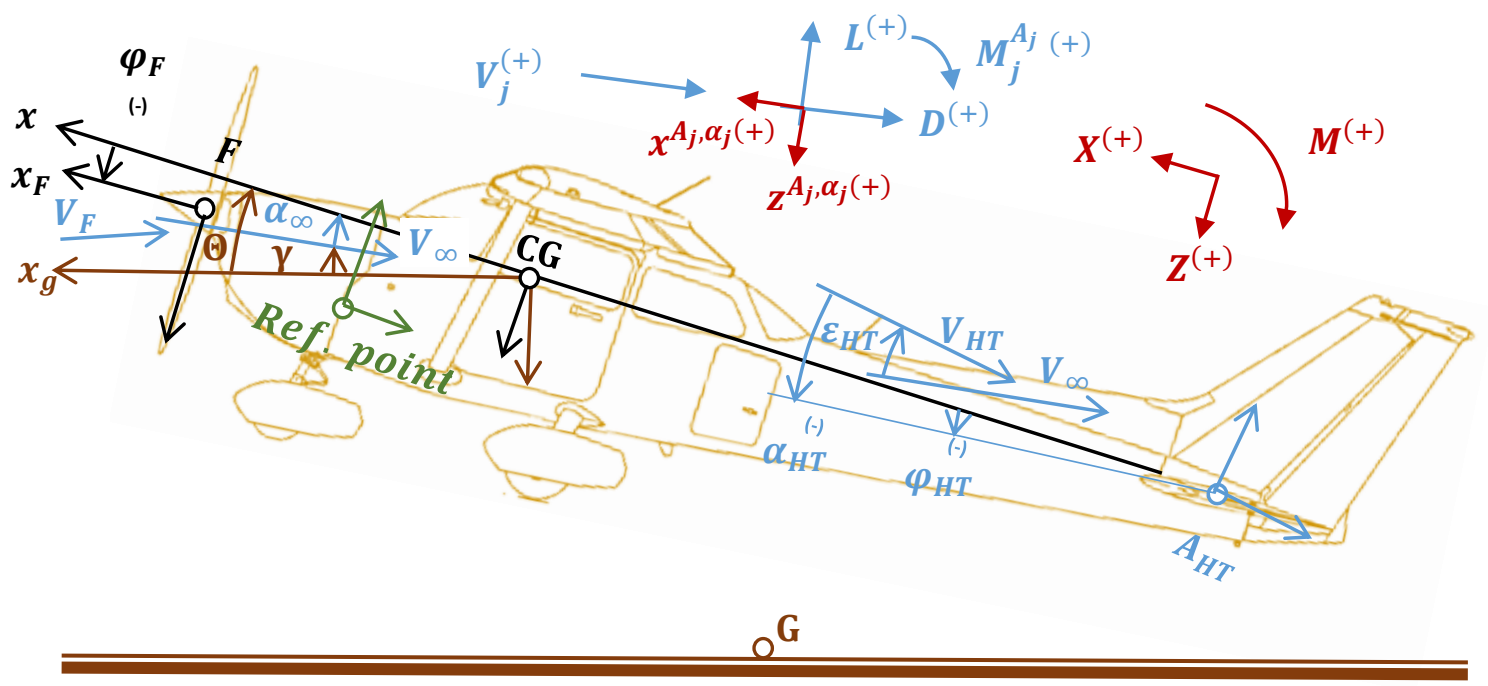

Figure 1: Axis systems and characteristic angles, mnemotechnic help in right up corner.

Angles and moments are controlled by right hand rule and dimensions and forces sign by axis system orientation. Plus (+) in upper index means the positive value of the vector in the picture, minus (-) means negative value of the vector in the picture. This mnemotechnic device helps to avoid mistake in derivation. Velocity in local air path axis system has opposite orientation, because we suppose, that the aircraft stands, and the air is moving. Lift and drag force have opposite orientation also, minus must be used in transformation. 


\subsection{Transformation}

C. G. is the center of rotation of an aircraft. Therefore, we need to transform all the dimensions, forces and moments from the local systems to body a. s. Transformation of the moment and forces is twostep process. Rotation of forces Figure 2 and movement of moment to C. G. Figure 3. In case, we need to transform against the arrow direction, opposite sign must be used in front of the angle or dimension value in transformation matrixes.

Note: If we transform from local air path system, we must use "minus" in front of Drag and Lift in force and moment transformation.

The schemes in the Figure 2 and Figure 3 was created as a mnemotechnic device, because there is high danger of making a mistake.

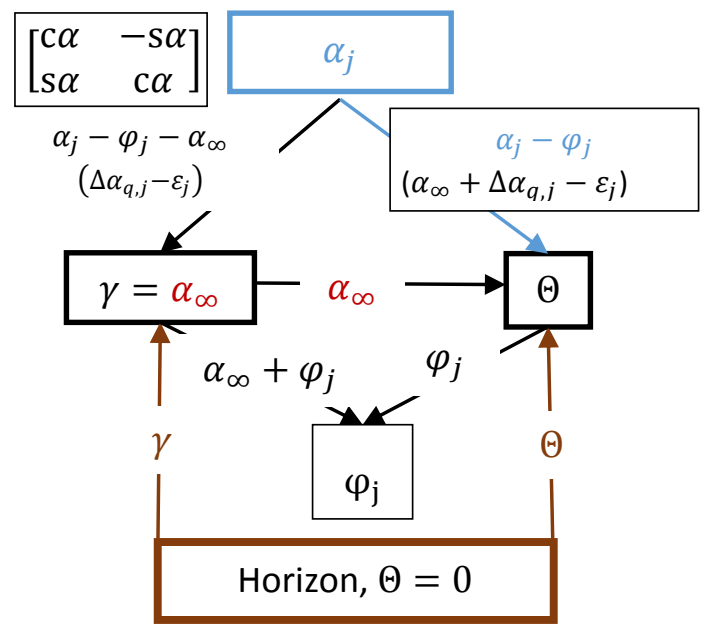

Force transformation from local air-path to body a.s.

$$
\left[\begin{array}{l}
X_{j} \\
Z_{j}
\end{array}\right]=\left[\begin{array}{cc}
\mathrm{c}\left(\alpha_{j}-\varphi_{j}\right) & -\mathrm{s}\left(\alpha_{j}-\varphi_{j}\right) \\
\mathrm{s}\left(\alpha_{j}-\varphi_{j}\right) & \mathrm{c}\left(\alpha_{j}-\varphi_{j}\right)
\end{array}\right]\left[\begin{array}{l}
-D_{j} \\
-L_{j}
\end{array}\right]
$$

Dimension transformation from body to flight path a.s.

$$
\begin{gathered}
{\left[\begin{array}{c}
x_{j}^{\gamma} \\
z_{j}^{\gamma}
\end{array}\right]=\left[\begin{array}{cc}
\mathrm{c}\left(-\alpha_{\infty}\right) & -\mathrm{s}\left(-\alpha_{\infty}\right) \\
\mathrm{s}\left(-\alpha_{\infty}\right) & \mathrm{c}\left(-\alpha_{\infty}\right)
\end{array}\right]\left[\begin{array}{l}
x_{j} \\
z_{j}
\end{array}\right]} \\
" j " \text { can be } \boldsymbol{F}, \boldsymbol{A}_{\boldsymbol{H} \boldsymbol{T}}, \boldsymbol{A}_{\boldsymbol{W}}, \ldots
\end{gathered}
$$

Figure 2: Rotation of the forces to different angle

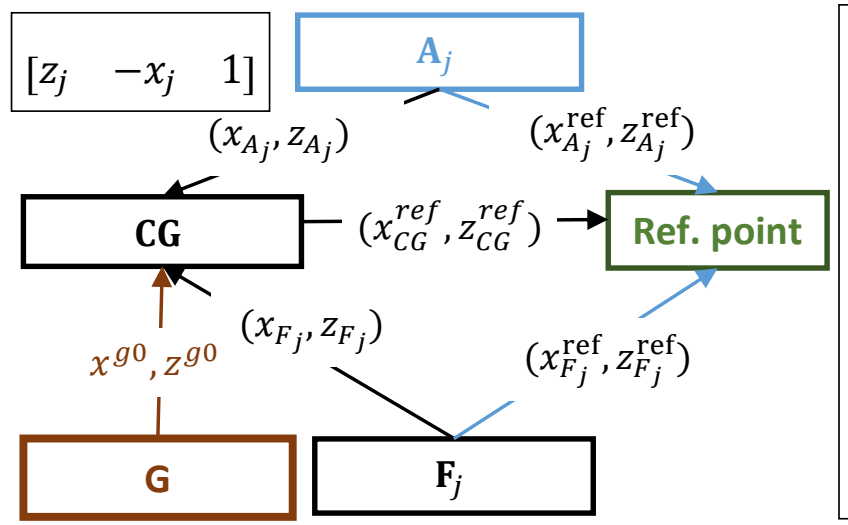

Moment transformation from local aerodynamic centre to the centre of gravity.

$$
\left[M_{j}\right]=\left[\begin{array}{lll}
Z_{j} & -x_{j} & 1
\end{array}\right]\left[\begin{array}{c}
X_{j} \\
Z_{j} \\
M_{j}^{A_{j}}
\end{array}\right]
$$

Dimensions and forces must have the same orientation. Most often it is $\Theta$.

Figure 3: Movement moment to different position

Because the centre of gravity can change a position in time, every point on the aircraft is better to define by the distance from reference point Figure 1. Positions of the points on the aircraft is possible to transform to body fixed axis system with origin in $C G$ and orientation of the $\mathrm{x}$ axis $\Theta$ :

$x_{j}=x_{C G}^{r e f}-x_{j}^{r e f} \quad$ oriented $\mathrm{x}$ position in body axis system.

$z_{j}=z_{C G}^{r e f}-z_{j}^{r e f} \quad$ oriented $z$ position in body axis system. 


\subsection{Airflow model}

Airflow model for longitudinal flight dynamics can be described by undisturbed dynamic pressure $p_{D}$ velocity $V_{\infty}$, AoA $\alpha_{\infty}$, and its changes as dynamic pressure change $k_{j}$, and airflow angle change $\varepsilon_{j}$. These changes can be caused by wing - horizontal tail interaction, propeller, wind gust etc. Dynamic pressure change affects a local velocity $V_{j}$. Airflow angle change $\varepsilon_{j}$ affects a local angle of attack $\alpha_{j}$.

In this study we use time delayed downwash angle and angular velocity influence on local velocity and AoA. These characteristics are not usually used, but it helps to avoid using other than local dynamic stability derivatives. $V_{j}$ and $\alpha_{j}$ are derived in following chapter.

\subsection{Dynamic stability derivatives avoiding: Downwash delay and angular velocity influence.}

Following part is showed on horizontal tail, but it can be general to all the aerodynamic parts on the aircraft, which can be replaced by the characteristics in one point.

Time angle of attack change will cause lift on the wing change. Time lift on the wing change will cause time downwash change at horizontal tail position. The change of downwash angle, due to lift, will affect the tail with $\Delta t_{\varepsilon}^{(+)} \cong-\frac{l_{\varepsilon}^{(-)}}{V_{\infty}}$ delay, because the horizontal tail is placed at given distance behind the wing. Figure 4

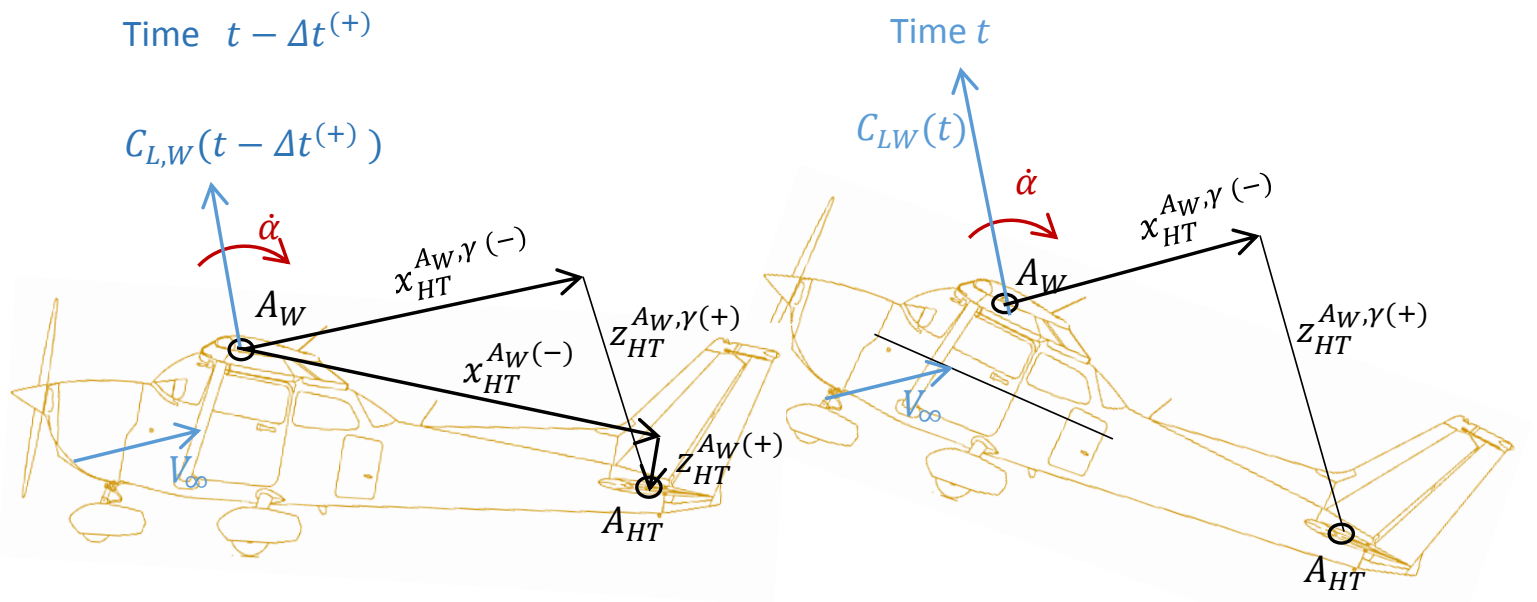

Figure 4: Time delayed downwash angle estimation

For explanation, very simple downwash model, which is useful for aspect ratio $>4$ and low AoA and short $z_{H T}^{A_{W}, \gamma}$, was chosen. For chosen model, downwash angle is a function of wing lift coefficient.

Exact time is not necessary and it's not easy to integrate. Delay of the downwash is approximately:

$$
\Delta t_{\varepsilon}^{(+)} \cong-\frac{x_{H T}^{A_{W}, \gamma(-)}}{V_{\infty}} \text {, where }\left[\begin{array}{c}
x_{H T}^{A_{W}, \gamma} \\
z_{H T}^{A_{W}, \gamma}
\end{array}\right]=\left[\begin{array}{cc}
\mathrm{c}\left(-\alpha_{\infty}\right) & -\mathrm{s}\left(-\alpha_{\infty}\right) \\
\mathrm{s}\left(-\alpha_{\infty}\right) & \mathrm{c}\left(-\alpha_{\infty}\right)
\end{array}\right]\left[\begin{array}{c}
x_{H T}^{A_{W}} \\
z_{H T}^{A_{W}}
\end{array}\right]
$$

Downwash is given by lift coefficient in $t-\Delta t_{\varepsilon}$ time.

$$
\varepsilon_{H T}=\varepsilon_{H T}\left(t-\Delta t_{\varepsilon}^{(+)}\right) \cong \frac{2 C_{L W}\left(t-\Delta t_{\varepsilon}^{(+)}\right)}{\pi A}
$$

Simulation needs saving of delayed characteristics (wing lift coefficient) during the simulation. 
Angular velocity causes change of angle of attack of the parts out of the rotation point (centre of gravity). Following figure and equations shows change of $\alpha_{H T}$ and $V_{H T}$ due to $q$.

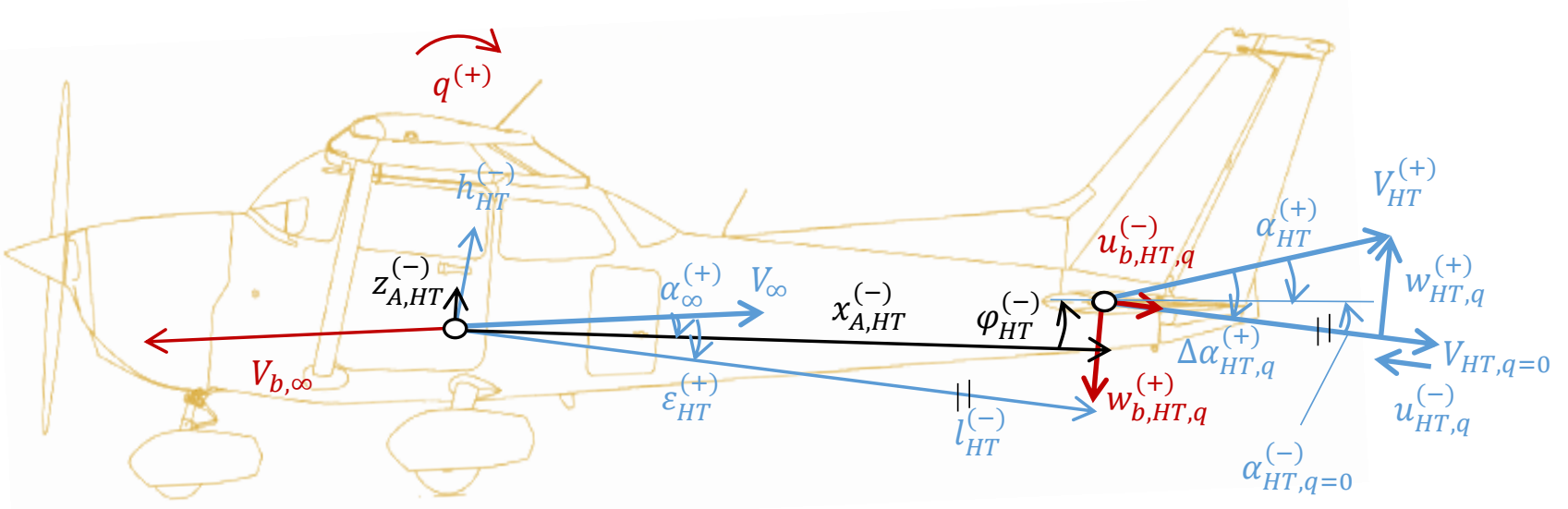

Figure 5: Angular velocity influence at the horizontal tail

We need to take characteristic of atmosphere at horizontal tail position at $\mathrm{q}=0$ :

$$
\varepsilon_{H T} \quad V_{H T, q=0}=\sqrt{2 \cdot k_{j} \cdot p_{D} / \rho_{j}} \cong \sqrt{k_{j}} V_{\infty}
$$

Transform dimensions to horizontal tail angle of attack:

$$
\left[\begin{array}{l}
l_{H T}^{(-)} \\
h_{H T}^{(+)}
\end{array}\right]=\left[\begin{array}{cc}
\mathrm{c}\left(-\left(\alpha_{\infty}-\varepsilon_{H T}\right)\right) & -\mathrm{s}\left(-\left(\alpha_{\infty}-\varepsilon_{H T}\right)\right) \\
\mathrm{s}\left(-\left(\alpha_{\infty}-\varepsilon_{H T}\right)\right) & \mathrm{c}\left(-\left(\alpha_{\infty}-\varepsilon_{H T}\right)\right)
\end{array}\right]\left[\begin{array}{c}
x_{A, H T}^{(-)} \\
z_{A, H T}^{(+)}
\end{array}\right]
$$

Compute velocity change due to angular velocity in case of moving body (index b):

$$
u_{b, H T, q}^{(-)}=q^{(+)} \cdot h_{q, H T}^{(-)} \quad w_{b, H T, q}^{(+)}=-q^{(+)} \cdot l_{q, H T}^{(-)},
$$

The red vectors in Figure 5 means moving aircraft body with some angular velocity. To transform the velocities to local air path a. s. we suppose not moving aircraft. The values will be the same, only the orientation in the picture is opposite (the blue vectors in the figure).

$$
u_{H T, q}^{(+)}=u_{b, H T, q}^{(+)} \quad w_{H T, q}^{(+)}=w_{b, H T, q}^{(+)} \quad V_{H T}=\sqrt{\left(V_{H T, q=0}+u_{H T, q}\right)^{2}+w_{H T, q}^{2}} \quad q_{H T}=q
$$

Finally, we compute angle of attack change at $A_{H T}$ position:

$$
\Delta \alpha_{H T}^{(+)}=\operatorname{asin} \frac{w_{q, H T}^{(+)}}{V_{H T}} \quad \alpha_{H T}=\alpha_{\infty}+\varphi_{H T}-\varepsilon_{H T}\left(t-\Delta t_{\varepsilon}\right)+\Delta \alpha_{H T, q}
$$

In case, $u_{H T, q} \ll V_{H T}$ and at low AoA, simple approximation can be used:

$$
V_{H T} \cong V_{H T, q=0} \quad \alpha_{H T} \cong \alpha_{\infty}+\varphi_{H T}-\varepsilon_{H T}\left(t-\Delta t_{\varepsilon}\right)+\left(-\frac{q \cdot x_{A_{H T}}}{V_{H T}}\right)
$$




\subsection{Aerodynamic forces in local air path axis system}

Part of an aircraft, which produces aerodynamic forces, as a wing, a fuselage, a horizontal tail, are usually described by aerodynamic coefficients. These coefficients can be a function of its angle of attack AoA, flap deflection $\delta$, Reynolds number $R e$, angular velocity $q$, Mach number $M$, and so on.

$$
C_{L, j}, C_{m, j}, C_{D, j}=f\left(\alpha_{j}, \delta_{j}, R e_{j}, M_{j}, q_{j}, \ldots\right)
$$

The model in this study allows to define the aerodynamic coefficients by linear function, general function or by a table of values. The reference point $A_{j}$ is usually close to aerodynamic centre of the part. Angle of attack $\alpha_{j}$ is referenced to the characteristic line of the part. It can be zero lift line or mean aerodynamic chord.

The forces in local air-path axis system Figure 6 can be described by these formulas.

$$
\begin{gathered}
p_{D, j}=k_{j} \cdot p_{D} \\
D_{j}=p_{D, j} \cdot C_{D, j} \cdot S_{j} \quad L_{j}=p_{D, j} \cdot C_{L, j} \cdot S_{j} \quad M_{j}^{A_{j}}=p_{D, j} \cdot C_{m, j} \cdot S_{j} \cdot c_{A, j}
\end{gathered}
$$

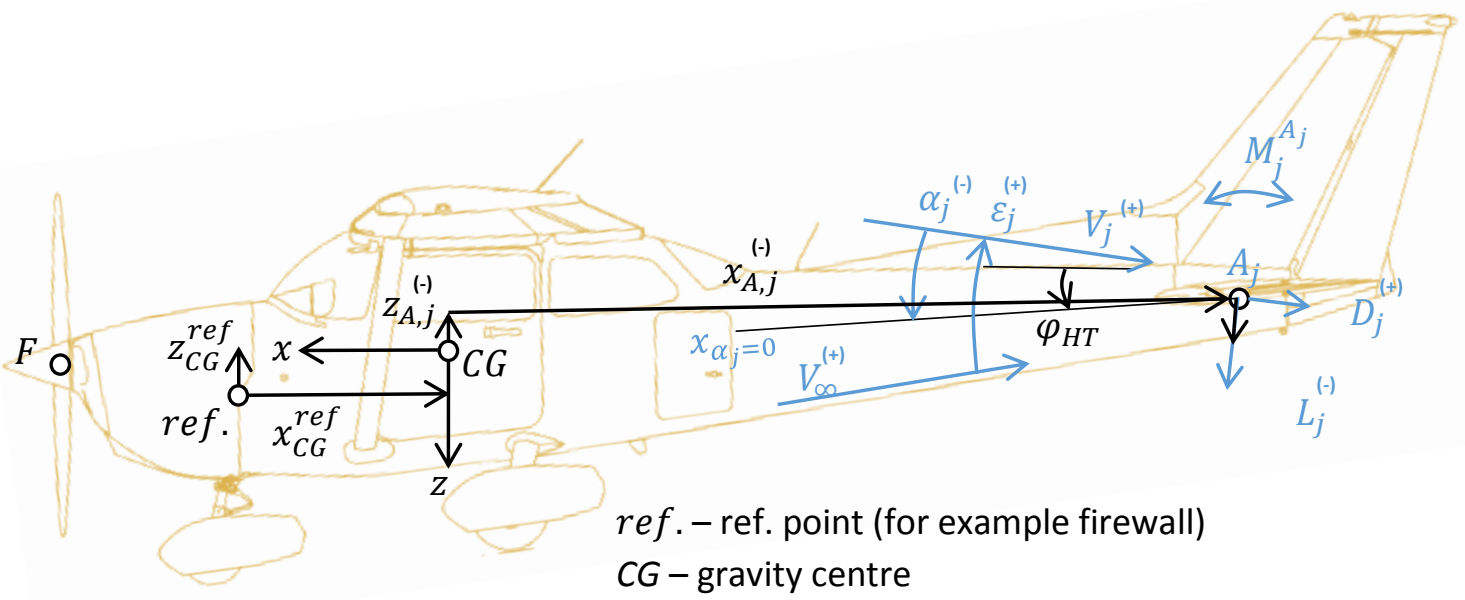

Figure 6: Local air-path a. s. definition on part $j=H T$

Lift and drag force have an opposite orientation, minus must be used in transformation therefore.

$$
\left[\begin{array}{l}
X_{j} \\
Z_{j}
\end{array}\right]=\left[\begin{array}{cc}
\mathrm{c}\left(\alpha_{j}-\varphi_{j}\right) & -\mathrm{s}\left(\alpha_{j}-\varphi_{j}\right) \\
\mathrm{s}\left(\alpha_{j}-\varphi_{j}\right) & \mathrm{c}\left(\alpha_{j}-\varphi_{j}\right)
\end{array}\right]\left[\begin{array}{l}
-D \\
-L
\end{array}\right] \quad M_{j}=M_{j}^{A}+\left(-D_{j}\right) \cdot z_{j}-\left(-L_{j}\right) x_{j}
$$

\subsection{Gravity forces}

Gravity force must be transformed to aircraft body fixed a. s. $X_{j}^{\Theta}=-m \cdot g \cdot \sin (\Theta), \quad Z_{j}^{\Theta}=m \cdot g$. $\cos (\Theta)$.

\subsection{Propulsion forces}

Propulsion AoA definition is $\alpha_{j}=\alpha_{\infty}+\varphi_{j}-\varepsilon_{j}+\Delta \alpha_{j, q}$, where $\Delta \alpha_{j, q} \cong-q^{(+)} \cdot x_{j}^{(+)} / V_{\infty}^{(+)}$.

In case of constant speed propeller, power available $P_{a, j}$ is approximately constant, thrust can be expressed as $X_{j}^{\varphi_{j}}=P_{a, j} / V$. Perpendicular force can be also important $Z_{j}^{\varphi_{j}}=-p_{D_{j}} \cdot C_{K}\left(\alpha_{j}\right) S_{j}$. Transformation to body fixed a. s. is necessary Figure 7. 


$$
\left[\begin{array}{l}
X_{j} \\
Z_{j}
\end{array}\right]=\left[\begin{array}{cc}
\mathrm{c}\left(-\varphi_{j}\right) & -\mathrm{s}\left(-\varphi_{j}\right) \\
\mathrm{s}\left(-\varphi_{j}\right) & \mathrm{c}\left(-\varphi_{j}\right)
\end{array}\right]\left[\begin{array}{c}
X_{j}^{\varphi_{j}} \\
Z_{j}^{\varphi_{j}}
\end{array}\right] \quad M_{j}=M_{j}^{F}+X_{j} \cdot z_{j}-Z_{j} \cdot x_{j}
$$

Figure 7: Propulsion force

\subsection{Equations of motion}

The forces, transformed to body fixed axis system, must be summed.

$$
X=\sum_{j} X_{j} \quad Z=\sum_{j} Z_{j} \quad M=\sum_{j} M_{j}
$$

For having simple equations of motion, we can transform summed forces to flight path a. s.:

$$
\left[\begin{array}{l}
X^{\gamma} \\
Z^{\gamma}
\end{array}\right]=\left[\begin{array}{cc}
\mathrm{c}\left(-\alpha_{\infty}\right) & -\mathrm{s}\left(-\alpha_{\infty}\right) \\
\mathrm{s}\left(-\alpha_{\infty}\right) & \mathrm{c}\left(-\alpha_{\infty}\right)
\end{array}\right]\left[\begin{array}{l}
X^{\Theta} \\
Z^{\Theta}
\end{array}\right]
$$

Note: Transformation to the flight path a. s. is convenient only for longitudinal equations of motions. For 6 DOF model, inertia moment would have to be transformed to flight path a. s. in every time step.

Equation of motion are derived from [10]. In every step we compute new velocity and flight path angle. Velocity perpendicular to flight path velocity is assumed to be zero.

$$
\begin{gathered}
\dot{V}=\frac{X^{\gamma}}{m} \quad \dot{\gamma}=-\frac{1}{V} \frac{Z^{\gamma}}{m} \quad \dot{q}=\frac{M^{C G}}{I_{y}} \quad \dot{\alpha}=q-\dot{\gamma} \quad(\dot{w}=\dot{\alpha} \cdot V) \quad(\dot{\Theta}=q) \\
\dot{x}_{g}=V \cdot \cos (\gamma) \quad \dot{z}_{g}=V \cdot \sin (\gamma) \quad\left(w_{i+1} \cong 0\right) \\
x_{i+1}=x_{i}+\dot{x}_{i+1} \Delta t \quad x \in V, q, \gamma, \Theta, x_{g}, z_{g} \quad \alpha_{\infty}=\Theta-\gamma \quad u=\left[\delta_{V}, \delta_{T}, \ldots\right]
\end{gathered}
$$

Even if first order numerical solver can be sufficient, fourth order Runge-Kutta solver with error estimation was chosen [10]. The simulation is two steps. Approximate error is determined by first simulation with rough time step. According to the error in rough simulation, the time step is refined.

\subsection{Trimming by bisection method with interval expansion}

Bisection method for trimming was not sufficient. Aircraft model parameters are changing during trimming process, so the solution can move out of the bisection interval. Bisection with interval expansion was developed therefore. Example of use is below.

We look for an angle of attack and we control the power of the engine and elevator deflection, so that forces and moment are at equilibrium. 
1. Choosing steady state flight initial conditions: $V_{r e f}, \gamma_{r e f}, \rho_{r e f}, m_{r e f}, x_{C G, r e f}, \delta_{T}$

2. Control parameters $\delta_{\text {HTref }}, P_{\text {ref }}$ can be zero.

3. Choosing precision conditions of trimmed state: $|X|<0.01 \mathrm{~N},|Z|<0.1 \mathrm{~N},|\mathrm{M}|<0.1 \mathrm{Nm}$

4. Choosing minimum and maximum $\alpha_{r e f}$ interval.

5. Computing required change in power and elevator deflection from the moment and $\mathrm{X}$ force.

$$
P_{i+1} \cong-X^{\gamma} \cdot V+P_{i}
$$

$$
Z_{H T_{i+1}} \cong \frac{M}{x_{A H T}}+Z_{H T_{i}} \quad \delta_{H T_{i+1}}=-\frac{\mathrm{Z}_{\mathrm{HT} i+1}}{p_{D H T} \cdot S_{H T} \cdot C_{L, H T_{\delta}}}
$$

The change in power is not exact, but it is enough for fulfilling the precision conditions.

6. Computing aircraft model for new initial power and elevator deflection.

7. The change in $\mathrm{Z}$ force result, from trimming process, is the condition for angle of attack variation.

8. If the difference of the change of $Z$ force is smaller than its magnitude $\left|Z_{\text {ref }, i+1}-Z_{\text {ref }, i}\right|<$ $\left|Z_{r e f, i}\right|$, it is possible, that the solution is out of examined interval. Expansion of the angle of attack interval is applied.

9. Otherwise bisection of the interval is applied.

Core part of Matlab code of bisection method with interval expansion is provided below:

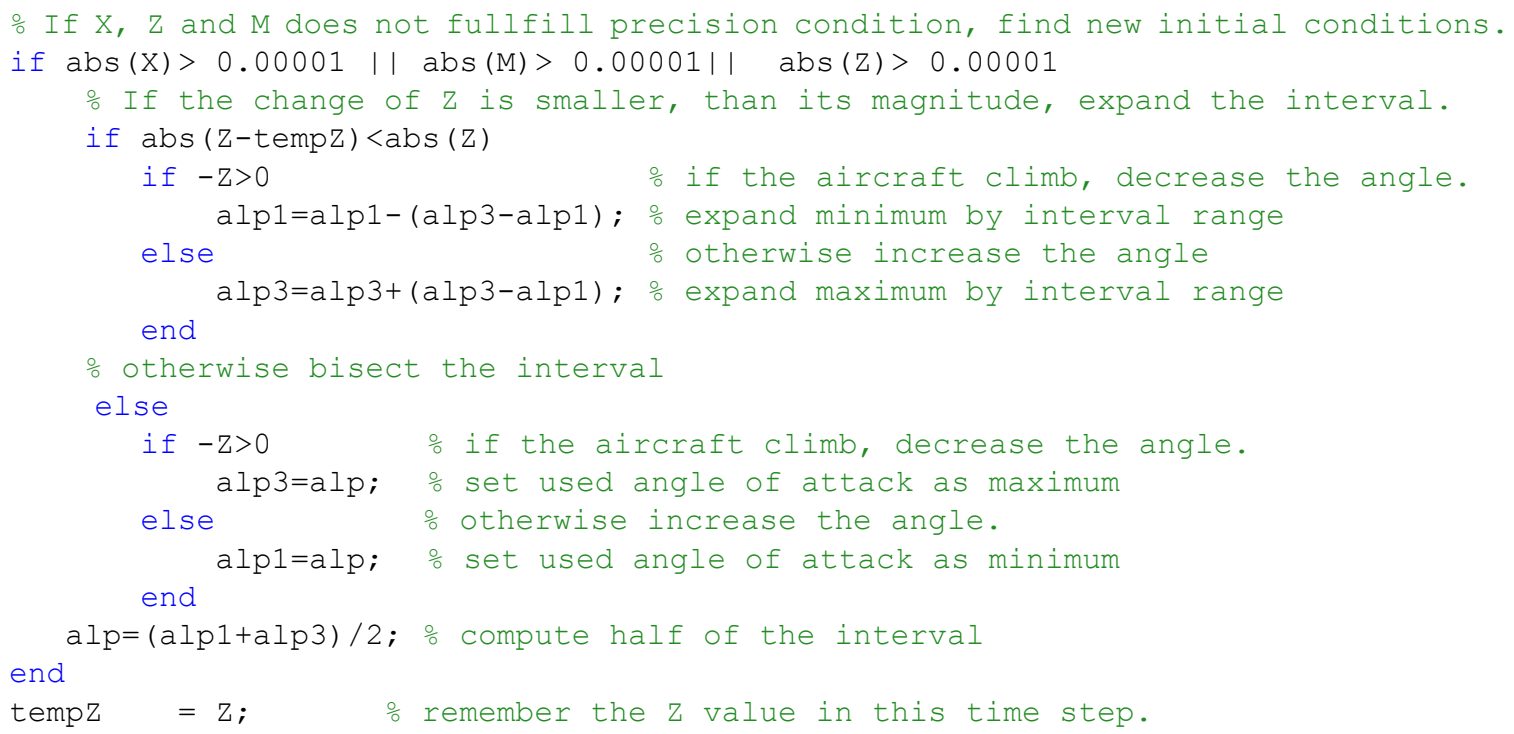

This trimming method is very fast. Only 37 iterations were necessary for steady state flight with maximally 1e-6 $\mathrm{N}$ parasitic forces in steady state. Two interval expansions were necessary during trimming process. Robustness of the method was not examined, but it works even for multidimensional trimming simultaneously. For example, finding the AoA and velocity, both by bisection with expansion method, for maximum thrust. Any other trimming algorithm can be used.

\section{LINEAR, NONLINEAR SIMULATION COMPARISON WITH EXPERIMENT}

Comparison of experiment with classic linear simulation and simple nonlinear simulation of the same model is presented in the Figure 8. It is a reaction of an aircraft to impulse to elevator deflection in $t=10 \mathrm{~s}$ taken out of experiment data. 

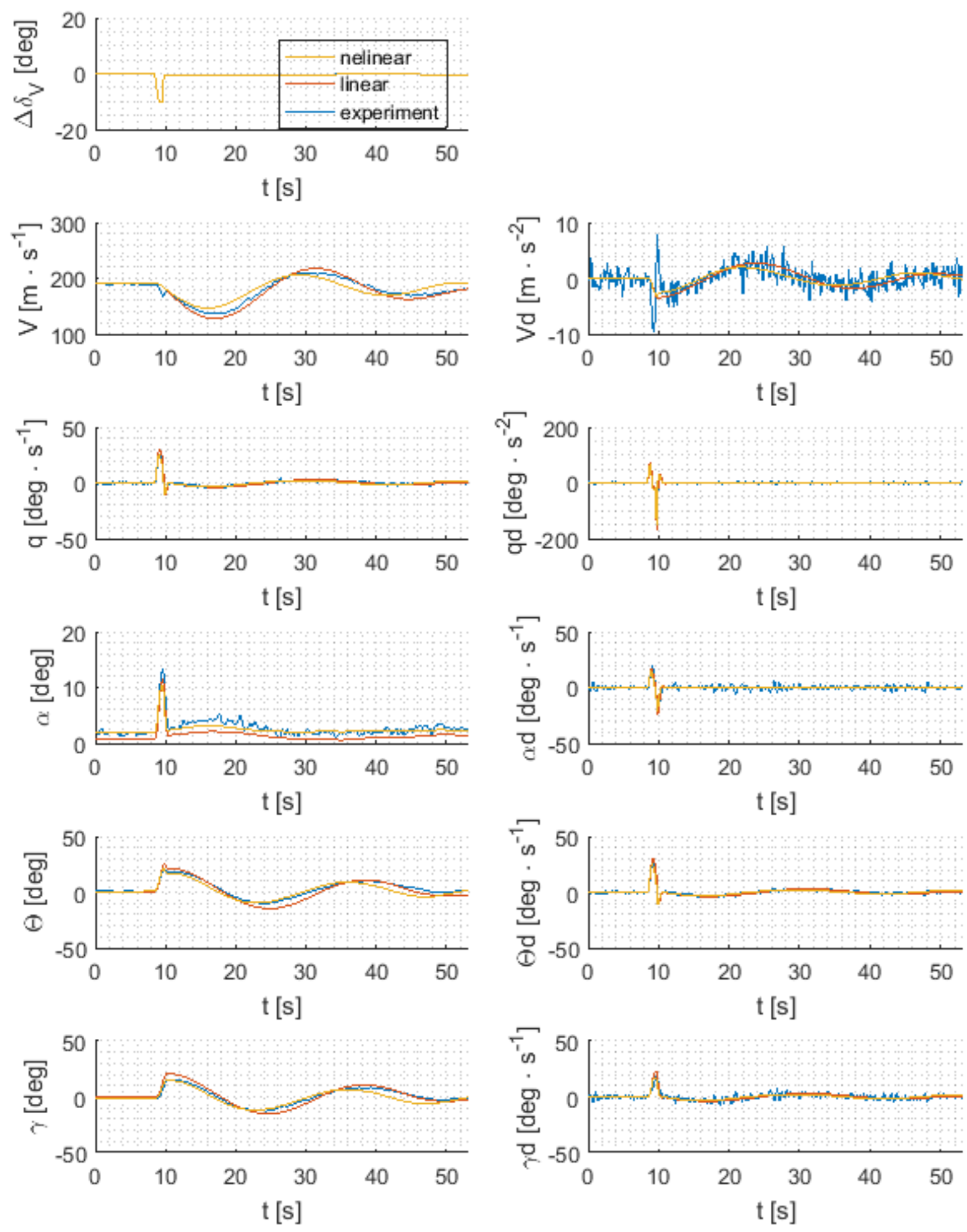

Figure 8: Comparison of experiment with linear and nonlinear simulation

Input characteristics of the models are the same and are derived from analytical methods [4]. Any of the models was not able to catch fast change of the velocity at the $10 \mathrm{~s} \mathrm{time.} \mathrm{Model} \mathrm{is} \mathrm{probably} \mathrm{too}$ simple to describe the phenomena. It can be caused by fuel swing from end to end or local stall during the maneuver on examined aircraft. Both simulations are close to the experiment. Linear simulation estimated better the velocity change. Non-linear estimated better the $\gamma, \Theta$ angle. The precision of the models is on the same level. 


\section{CONCLUSION}

The paper describes alternative non-linear simulation of an aircraft and compare it to the linear and an experiment. The precision of the method is comparable to the linear simulation on examined case. Non-linear simulation allows easier modifications of the model. Non-linear coefficients can be included. Simplification of the model is not necessary compared to classic theory. The physics of the model is more obvious, and number of required equations is low. Disadvantage is iterative trimming of an aircraft. Following problems were solved. Dynamic stability derivatives were succeeded by angle of attack modification with angular velocity and by delayed downwash model implementation. Bisection with interval expansion was used for trimming process. Time step refinement was used in the simulation.

Non-linear simulation of the whole aircraft is well known nowadays [11], but it is not usually used at the very beginning phase of conceptional design. Flight stability and control is not solved in the first part of conceptual design very often because it is too complex process. It was shown in introduction chapter, that linear simulation can be very simple process, can be finished in the very first part of conceptional design. Other chapters show, that non-linearities can be included quite easily.

\section{ACKNOWLEDGEMENTS}

The research leading to these results has received funding from the Ministry of Education, Young and Sport under the National Sustainability Programme I (Project LO1202).

\section{REFERENCES}

[1] BRYAN, G. H., (1911). Stability in aviation: an introduction to dynamical stability as applied to the motions of aeroplanes. London: Macmillan and co., limited.

[2] Finck, R. D. (1978). USAF (United States Air Force) Stability and Control DATCOM (Data Compendium). MCDonnell Aircraft Co St Louis Mo.

[3] Gato, William, et al. (2015). Flight simulation modeling of aircraft dynamic stall aerodynamics. U.S. Patent Application No. 14/823,507.

[4] Daněk, Vladimír, (2011). Mechanika letu. Brno: Akademické nakladatelství CERM.

[5] Phillips, Warren F. (2010). Mechanics of flight. 2nd ed. Hoboken, N.J.: J. Wiley

[6] Cook, M. V. (2012). Flight dynamics principles: a linear systems approach to aircraft stability and control. Butterworth-Heinemann.

[7] Etkin, B., \& Reid, L. D. (1996). Dynamics of flight: stability and control (Vol. 3). New York: Wiley.

[8] Kostić, Ivan, et al. (2013). Hybrid approach in the initial aerodynamic, stability and performance calculations of a light airplane. Tehnički vjesnik.

[9] Kostić, Ivan A., Zoran A. Stefanović, and Olivera P. Kostić. (2014) Aerodynamic analysis of a light aircraft at different design stages. FME Transactions.

[10] Stevens, B. L., et al. (2015). Aircraft control and simulation: dynamics, controls design, and autonomous systems. John Wiley \& Sons.

[11] Garza, F. R., \& Morelli, E. A. (2003). A collection of nonlinear aircraft simulations in matlab.

[12] DATA SHEETS. Aerodynamics, Vol. I, II a III. Royal Aeronautical Society (UK), 1965. 


\section{NOMENCLATURE}

AoA, $\alpha \quad$ angle of attack

$A \quad$ local air-path a. s. center or aspect ratio.

$e \quad$ Oswald efficiency number

$b \quad$ wing span or body width

CFD Computational Fluid Dynamics

$C G \quad$ Centre of gravity

$C_{L}, C_{D}, C_{m}$ lift, drag and moment coefficient

c reference dimension of coefficient, cosine

a. s. axis system

G reference ground point

$k_{H T} \quad$ dynamic pressure reduction at HT

$\tilde{l}_{H T} \quad=-x_{H T}$ distance of HT behind CG

$\mathrm{m} \quad$ weight

M moment, Mach number

$P_{a} \quad$ power available

$p_{D} \quad$ dynamic pressure

Re Reynolds number

$F \quad$ reference thrust point

$S \quad$ reference area of the coefficient

$\mathrm{s} \quad$ sinus

V velocity

VLM Vortex Lattice Method

$\mathrm{x}, \mathrm{z}, \alpha \quad$ oriented position and angle

$\mathrm{u}, \mathrm{w}, \mathrm{q} \quad$ velocities components

$X, Z, M \quad$ forces and moment

or "d" time derivative $\alpha \quad$ angle of attack

$\overline{\dot{\alpha}}=\dot{\alpha} c_{A} / 2 \mathrm{~V}$ nondimensional AoA time change

$\bar{q}=q c_{A} / 2 V$ nondimensional $q$ time change

$\gamma \quad$ flight path angle

$\delta \quad$ deflection, control variable

$\varepsilon \quad$ downwash angle

$\varphi \quad$ incidence angle

$\Theta \quad$ pitch angle

$\rho \quad$ air density

$0 \quad$ coefficient value at given angle equal 0

A aerodynamic

air airflow

B body

CG center of gravity

elevator

fuselage

dimensions in moving normal earth a. s.

trimming iteration

any aerodynamic part

thrust force

horizontal tail

root

St time and time increment

wing

freestream characteristic

$K_{W} \quad$ Correction factor of wing position. Typically, high-wing monoplane 0.95, low-wing 1.1.

$K_{A B} \quad$ Correction factor of aerodynamic center shift due to body [12].

$K_{B} \quad$ Correction factor of dynamic stability derivative due to body. Typical value is $1.1 \div 1.2$ for classic conception aircraft. It is derived from horizontal tail dynamic derivatives correction [4]

\section{COPYRIGHT STATEMENT}

The authors confirm that they, and/or their company or organization, hold copyright on all of the original material included in this paper. The authors also confirm that they have obtained permission, from the copyright holder of any third party material included in this paper, to publish it as part of their paper. The authors confirm that they give permission, or have obtained permission from the copyright holder of this paper, for the publication and distribution of this paper as part of the READ 2018 proceedings.

This is an open access article distributed under the Creative Commons Attribution License which permits unrestricted use, distribution, and reproduction in any medium, provided the original work is properly cited. (CC BY 4.0). 